\title{
NLC Positron Production Target
}

\author{
RECEIVED \\ APR 151996 \\ O.E. Krivosheev and N.V. Mokhov \\ OSTI \\ Fermi National Accelerator Laboratory \\ P.O. Box 500, Batavia, Illinois 60510
}

S.I. Striganov

Institute for High Energy Physics

Protvino, 142284, Russia

March 1996

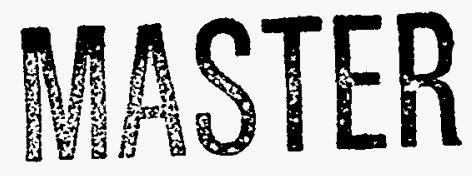

Operated by Universities Research Association Inc. under Contract No. DE-ACO2-76CHO3000 with the United States Department of Energy 


\section{Disclaimer}

This report was prepared as an account of work sponsored by an agency of the United States Government. Neither the United States Government nor any agency thereof, nor any of their employees, makes any warranty, express or implied, or assumes any legal liability or responsibility for the accuracy, completeness, or usefulness of any information, apparatus, product, or process disclosed, or represents that its use would not infringe privately owned rights. Reference herein to any specific commercial product, process, or service by trade name, trademark, manufacturer, or otherwise, does not necessarily constitute or imply its endorsement, recommendation, or favoring by the United States Government or any agency thereof. The views and opinions of authors expressed herein do not necessarily state or reflect those of the United States Government or any agency thereof. 


\title{
NLC Positron Production Target
}

\section{O. E. Krivosheev, N. V. Mokhov and S. I. Striganov ${ }^{+}$ \\ Fermi National Accelerator Laboratory}

P.O. Box 500, Batavia, Illinois 60510

+Institute for High Energy Physics, Protvino, 142284, Russia

\section{March 28, 1996}

\begin{abstract}
The NLC positron production target is optimized with respect to positron yield, target integrity, cooling and shielding.
\end{abstract}




\section{Introduction}

The proposed Next Linear Collider (NLC) is a $e^{+} e^{-}$linear collider with the c. m. energy up to $1.5 \mathrm{TeV}$ [1]. One of the essential NLC components is a target used for producing a low energy positron beam to be captured and accelerated. The positron production target design should take into account and properly balance the positron yield per initial electron, target integrity, shielding and thermal problems. A series of Monte Carlo simulations were performed for the various target materials and configurations. The EGS-4 [2], GEANT-3 [3] and MARS [4] Monte Carlo codes were used in the calculations.

\section{Design Parameters}

The positron yield calculations were performed for the SLAC electron beams with the parameters presented in Table 1 [1].

Table 1: Electron Beam Parameters.

\begin{tabular}{|l|r|r|}
\hline Parameter & NLC $500 \mathrm{GeV}$ & NLC 1.0 or $1.5 \mathrm{TeV}$ \\
\hline Energy $\mathrm{E}_{e^{-}}, \mathrm{GeV}$ & 3.11 & 6.22 \\
\hline Intensity, $N_{e^{-}} /$bunch & $1.50 \cdot 10^{10}$ & $1.50 \cdot 10^{10}$ \\
\hline Bunch length, psec & 5 & 5 \\
\hline N bunches $/$ pulse & 90 & 75 \\
\hline Intensity, $N_{e^{-}} /$pulse & $1.35 \cdot 10^{12}$ & $1.13 \cdot 10^{12}$ \\
\hline Rep. rate $(\mathrm{Hz})$ & 180 & 120 \\
\hline Beam Power $(\mathrm{kW})$ & 121 & 134 \\
\hline
\end{tabular}

Positrons generated in the target have to be captured into a flux concentrator with a relatively small spatial acceptance (from 3 to $5 \mathrm{~mm}$ ) and narrow energy bin of 5 to $25 \mathrm{MeV}$. The positron bunch accelerated in the linac goes finally to the collider. The design goal is to achieve a yield of approximately 1 positron per electron at the exit from the flux concentrator.

\section{Positron Yield}

The calculations have been performed for the electron beam parameters given in the last column of Table 1. It is assumed that the initial beam has a Gaussian spatial distribution with $\sigma_{x}=\sigma_{y}=0.8 \mathrm{~mm}$, a Gaussian energy distribution with $\sigma_{E}=0.001 \times \mathrm{E}_{e^{-}}$ and a Gaussian angular distribution with $\sigma_{\theta}=5 \mathrm{mrad}$. Figure 1 shows the absolute yield (in terms of the number of $e^{+}$per initial electron) for a solid tungsten target, $4 L_{R}$ thick and $100 \mathrm{~cm}$ in radius, for several collection radii $R_{c o l}$. 

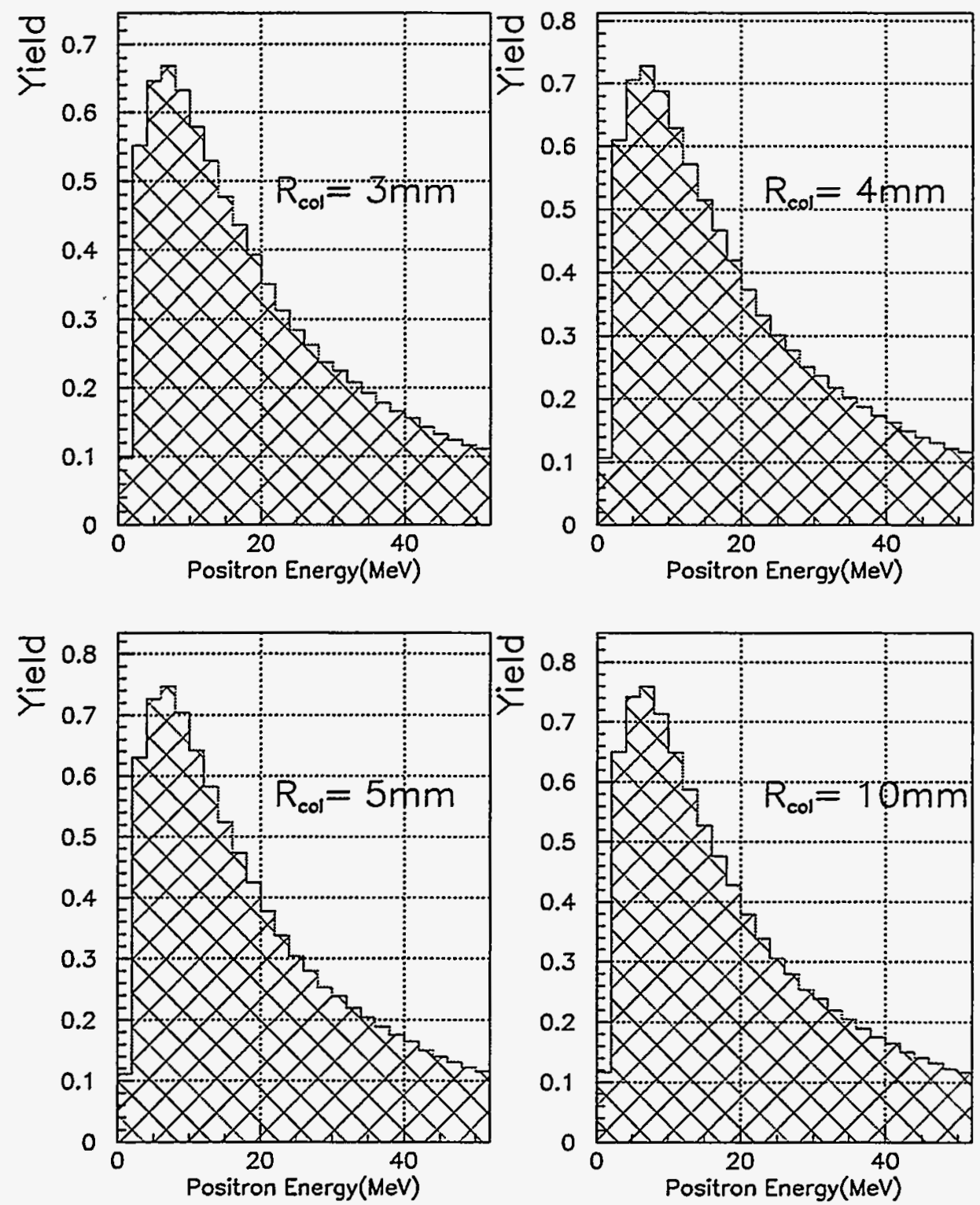

FIGURE 1. Absolute positron yield per $\triangle \mathrm{E}=2 \mathrm{MeV}$ per one initial electron for the solid tungsten target, $\mathrm{E}_{e^{-}}=6.22 \mathrm{GeV}$.

Taking into account a flux concentrator collection efficiency equal to $40 \%$, a solid tungsten target gives a yield of about 1.9 positrons per electron, which exceeds the design goal [1]. At the same time, there are severe problems with tungsten as a material for the target. It is fragile and has low specific heat and thermal conductivity, that leads to certain mechanical and cooling problems. Some mitigation is possible with tungsten alloys, but one can try copper instead. Its relatively low $Z$ can result in somewhat decreased positron yield, but copper is far superior from the mechanical and thermal standpoints. A few runs were performed for several copper targets: cylinders and cones of different radii. 

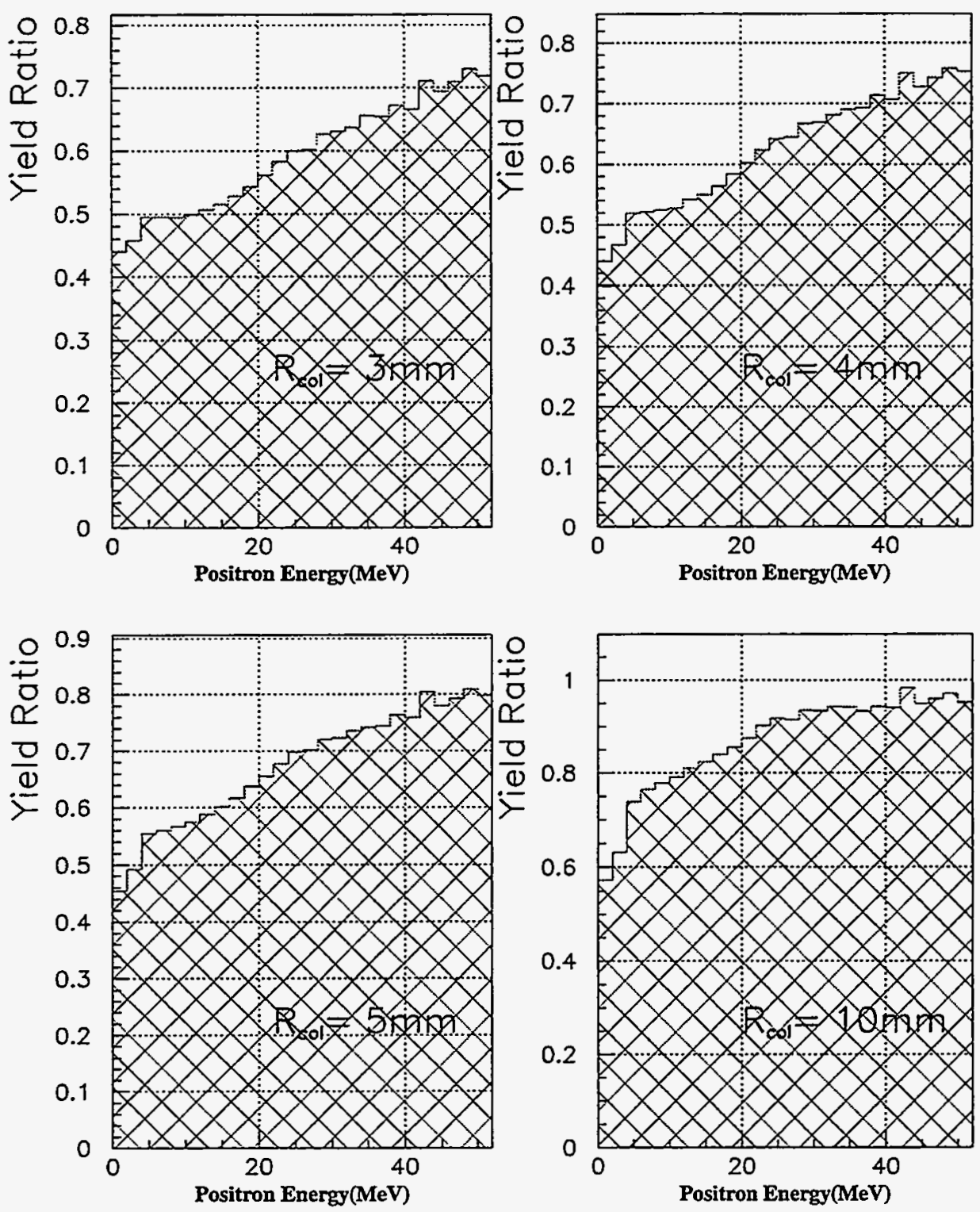

FIGURE 2. Ratio of positron yield per $\triangle \mathrm{E}=2 \mathrm{MeV}$ from copper target to that from tungsten. Target thicknesses are $4 L_{R}$ and radii are $3 \mathrm{~mm}$.

Figures 2 and 3 show that the positron yield from copper is about $60 \%$ of that for tungsten in the energy interval 5 to $25 \mathrm{MeV}$, i. e. about $1.1-1.2 e^{+}$per electron after the same flux concentrator. A yield enhancement can be achieved by using the wire-like cylinder or cone target and collecting positrons in a wider spatial and energy bins. These techniques were proposed in [5] and need additional calculation and design efforts for a nonstandard capture and concentrator system. 

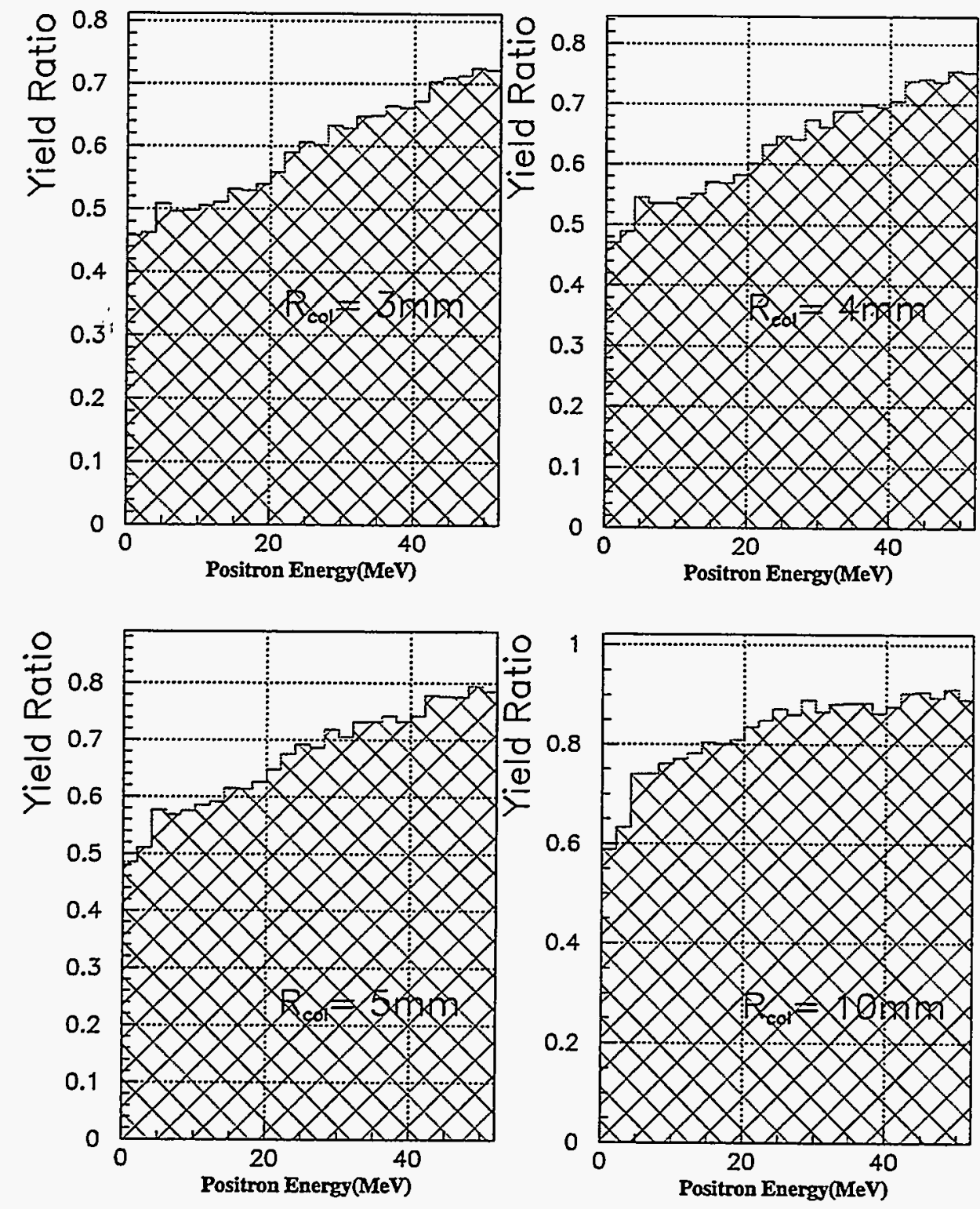

FIGURE 3. Ratio of positron yield from cone copper target per $\triangle E=2 \mathrm{MeV}$ to that from tungsten. Target thicknesses are $4 L_{R}$, cone upstream radius is $4 \mathrm{~mm}$ and downstream radius is $1 \mathrm{~mm}$.

\section{Thermal and Integrity Analyses}

Energy deposition density in tungsten and copper targets $6 \mathrm{~mm}$ in diameter is shown in Figures 4 and 5, respectively. One can see that the copper target has less energy deposited per unit volume. On the other hand, integral energy depositions are rather close to each other for the same target lengths as shown in Table 2. 
a
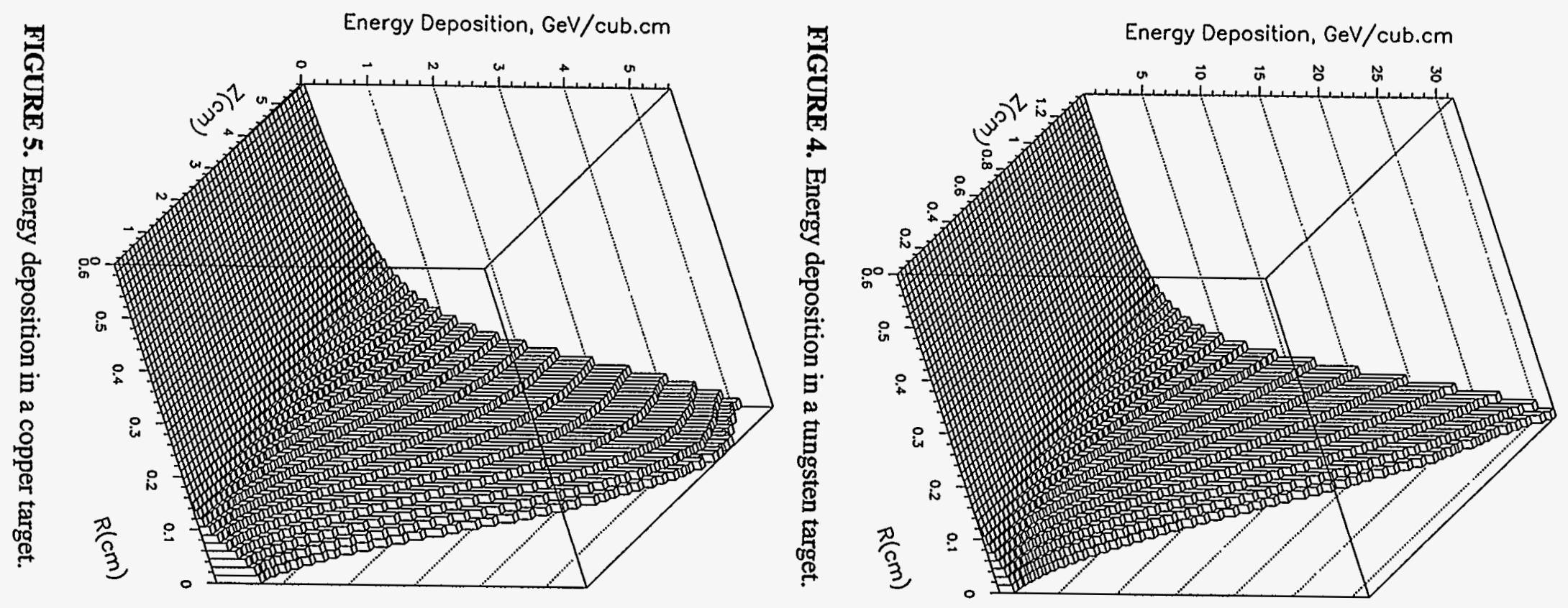
Table 2: Fraction of the beam energy (\%) deposited in tungsten and copper cylindrical targets of different lengths $L$ (in units of the radiation length $L_{R}$ ). Last column shows the peak energy deposition density $\epsilon_{\max }(J / g)$.

\begin{tabular}{|l|l|l|l|l|l|}
\hline$L$ & 2 & 4 & 6 & 8 & $\epsilon_{\max }$ \\
\hline $\mathrm{W}$ & 2.5 & 15.0 & 36.1 & 56.9 & 174 \\
\hline $\mathrm{Cu}$ & 4.2 & 21.7 & 46.5 & 67.6 & 90 \\
\hline
\end{tabular}

The peak energy deposition density in the tungsten targets is very close to the shock wave limit, which is $200 \mathrm{~J} / \mathrm{g}$ in this material. For this reason the SLAC team suggests special mitigating measures in order to prevent target destruction, namely, a spoiler to increase the initial beam spot size and a target rotation with relatively high speed.

Contrary, in copper the peak energy density is about $20 \%$ of the corresponding shock wave limit, that can drastically simplify the target operation. With the current proposal, the tungsten target provides about $40 \%$ higher positron yield, with no margin for further increasing of the production rate. At the same time, copper has at least a factor of five margin which allows increasing the positron yield just by using more intense electron beam.

Thermal analysis was performed using the ANSYS code [6]. The effective cooling system with $\Delta T=0$ at the target outer radius was assumed. Figure 6 shows a twodimensional maximum temperature distribution in the copper target $0.4 \mathrm{sec}$ after the beginning of the irradiation. Figure 7 shows the time dependence of maximum temperature in the copper target with a steady-state regime reached after about $0.3 \mathrm{sec}$. One can see that with a good cooling system the maximum temperature in the target is $\approx 700^{\circ} \mathrm{C}$, that allows one, in principle, to stay with a stationary target.
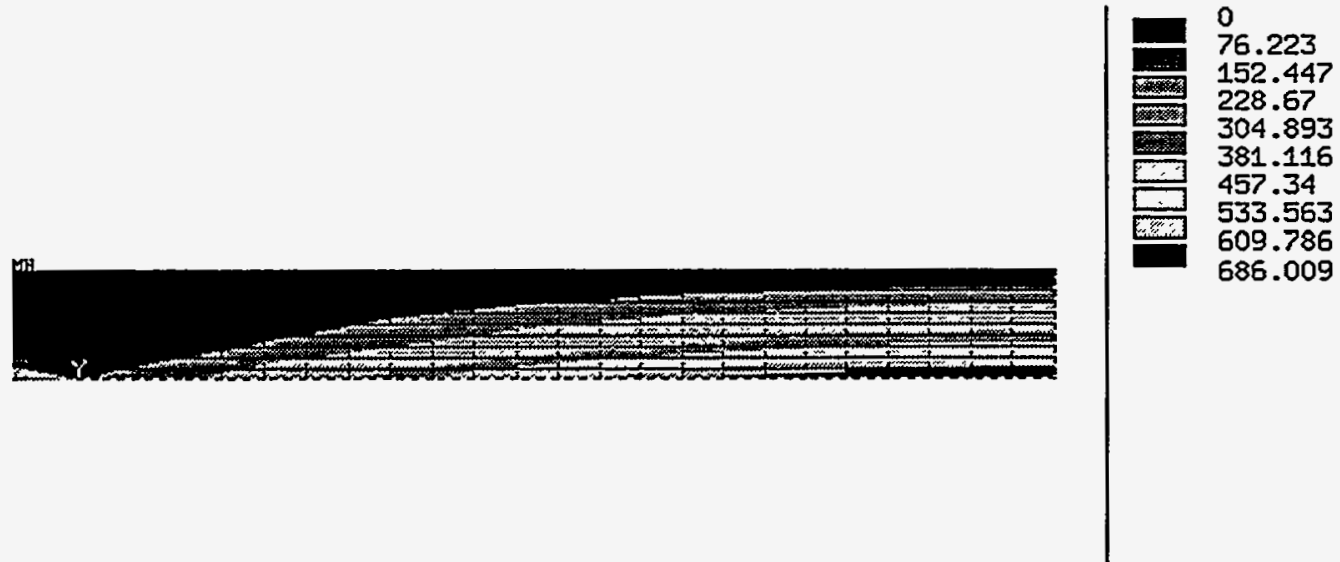

FIGURE 6. Temperature distribution after the pulse. 


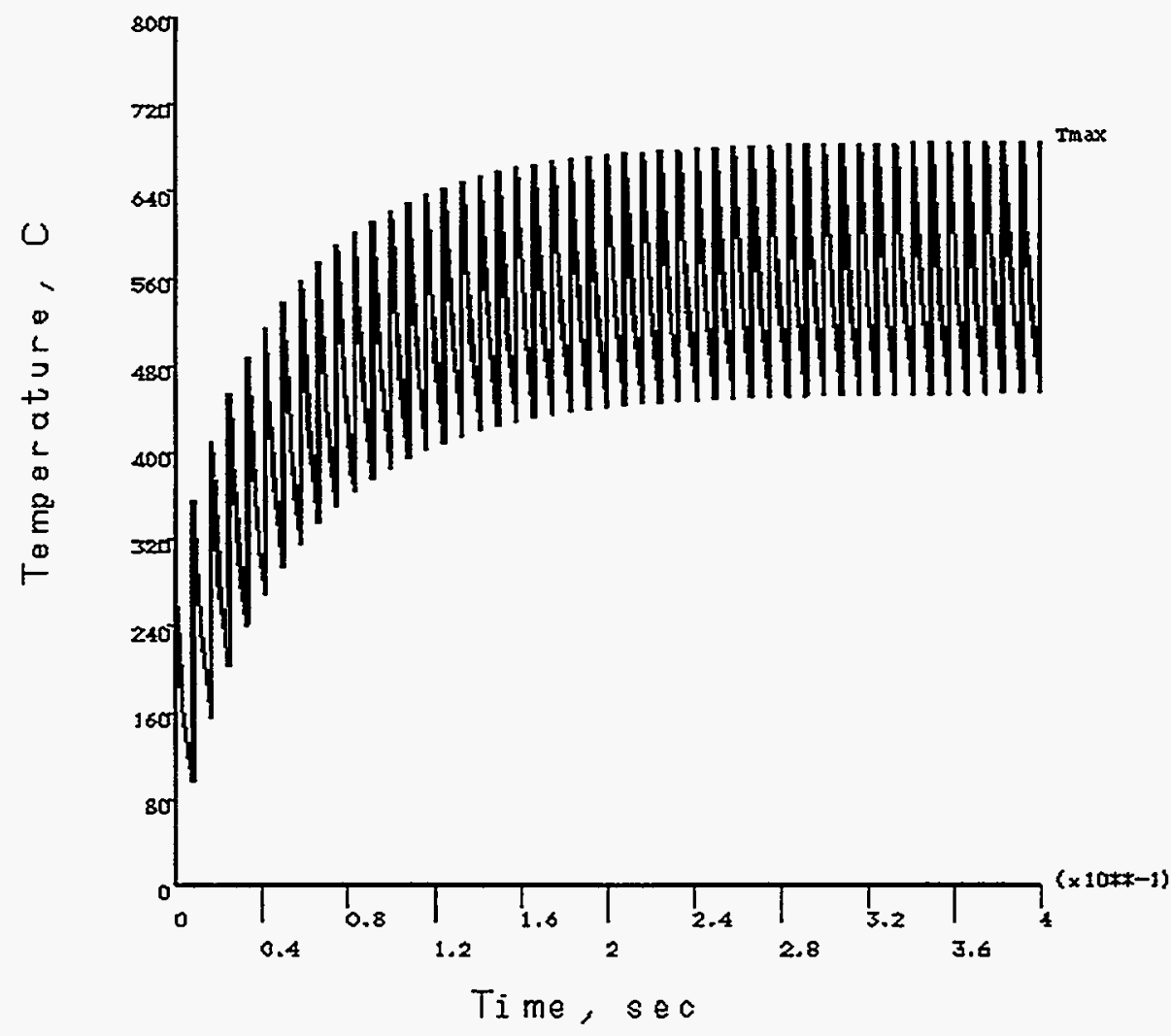

FIGURE 7. Maximum temperature in the copper target as a function of time.

\section{Shielding}

A copper target has another advantage, lower neutron yield, which reduces the required shielding. Calculated with the MARS code, hadron (E>14MeV) and lowenergy neutron $(\mathrm{E}<14 \mathrm{MeV})$ fluxes around tungsten and copper targets are presented in Table 3. The neutron yield is almost 6 times lower for the copper target, that results in a more compact target zone shielding. Estimated residual dose rate for the copper target is lower compared to the tungsten one.

Table 3: Particle flux around tungsten and copper targets ( $4 L_{R}$ long and $6 \mathrm{~mm}$ diameter) irradiated with $6.22 \mathrm{GeV}$ electron beam of $1.13 \times 10^{12} \mathrm{ppp}$ at $120 \mathrm{~Hz}$ rate.

\begin{tabular}{|l|l|l|}
\hline Target & $\Phi_{n}\left(\mathrm{~cm}^{-2} \mathrm{~s}^{-1}\right)$ & $\Phi_{h}\left(\mathrm{~cm}^{-2} \mathrm{~s}^{-1}\right)$ \\
\hline $\mathrm{W}$ & $6.25 \cdot 10^{12}$ & $4.72 \cdot 10^{11}$ \\
\hline $\mathrm{Cu}$ & $1.04 \cdot 10^{12}$ & $4.39 \cdot 10^{11}$ \\
\hline
\end{tabular}




\section{Conclusions}

Copper is suggested as a material for the positron target with yield of $\geq 1$ positron per electron. In contrast to tungsten, copper is farther from the shock-wave limit and has much better thermal properties. A copper target can be designed as a stationary unrotated cylinder with intensive cooling. With a copper target it is possible to increase further positron yield using a more intense $e^{-}$beam. Hadron and lowenergy neutron yield is lower for the copper target in comparison with the tungsten one, reducing the volume of the required shielding.

\section{Acknowledgments}

The authors wish to thank Mike Foley, Bruce Hoffman and Mark Reichanadter for their help with the ANSYS code and Jim Holt for useful discussions.

\section{References}

[1] “NLC Zero Design Report, March Workbook” (1995).

[2] W. Nelson, H. Hirayama and D. Rogers, "The EGS-4 Code System", SLAC Report-265 (1985).

[3] "GEANT, Detector Description and Simulation Tool", CERN, Geneva (1994).

[4] N. V. Mokhov, "The MARS code system Users Guide, version 13(95)", Fermilab-FN-628 (1995).

[5] R. Donahue and W. Nelson, "Alternative Positron Target Design for ElectronPositron Colliders", SLAC-Pub-5702 (1991).

[6] “ANSYS User's Manual”, Vol. I and II, SASI Publications (1994). 


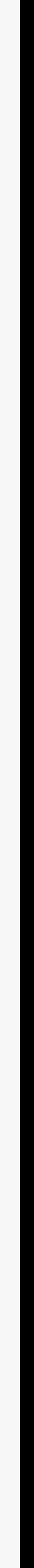

